\title{
Sex-specific association between infant diet and white matter integrity in 8-y-old children
}

\author{
Xiawei Ou ${ }^{1,2,3}$, Aline Andres ${ }^{1,2}$, Mario A. Cleves ${ }^{1,2}$, R.T. Pivik ${ }^{1,2}$, Jeffrey H. Snow ${ }^{1,2}$, Zhaohua Ding $^{4}$ and Thomas M. Badger ${ }^{1,2}$
}

BACKGROUND: The American Academy of Pediatrics recommends breastfeeding, which is well known to promote cognitive and behavioral development. The evidence for why this occurs is not well understood.

METHODS: Fifty-six 7.5- to 8.5-y-old healthy children were breastfed (BF; $n=22,10$ males) or formula-fed (FF; $n=34,16$ males) as infants. All children were administered: the Reynolds Intellectual Assessment Scale (RIAS); the Clinical Evaluation of Language Fundamentals (CELF-4) tests; and magnetic resonance imaging of the brain. Diffusion tensor imaging (DTI) measured fractional anisotropy (FA) values were correlated with RIAS and CELF-4 scores.

RESULTS: DTI tract-based spatial statistics (TBSS) analyses showed multiple white matter regions in the left hemisphere with significantly higher FA ( $P<0.05$, corrected) values in BF than FF males, but no significant group differences in females. Males who were exclusively BF for at least 1 y appeared to have the greatest differences in FA. Mean FA values positively correlated with composite scores of RIAS $(P=0.03)$ and CELF-4 $(P=0.02)$.

CONCLUSION: Breastfeeding during infancy was associated with better white matter development at 8 y of age in boys. A similar association was not observed in girls.

B reast milk contains what most pediatricians and nutritionists consider to be nature's most complete diet for newborn infants. While having the known major nutrients (proteins, lipids, carbohydrates, vitamins, and minerals) required for "normal" infant growth, formulas lack specific factors that promote human development to the same level as breast milk (1). The importance of breast milk for brain development is particularly highlighted in studies of children born preterm, where significant advantages in neurodevelopment are gained in those fed breast milk during infancy as compared to formula $(2,3)$. This is because approximately one-third of brain growth occurs in the last $6-8 \mathrm{wk}$ of gestation and breast milk provides a unique mixture of nutrients and bioactive compounds which better support the "extra-utero" brain development of preterm babies than commercially available formulas (4).
Less is known about the influence of diet and nutritional status on brain development and cognitive function in the first year of life of healthy full-term infants. However, better scores on standardized behavioral tests of infant development have been reported in breastfed (BF) than formula-fed (FF) fullterm infants (5). Positive associations between breastfeeding and higher intelligence quotient (IQ), better school performance, and greater neurocognitive development have been observed in numerous studies (6-8). Furthermore, the duration and exclusiveness of breastfeeding appear to be positively correlated with IQ and academic achievement $(7,9)$. Clinical studies have revealed that breastfeeding is associated with larger head circumference and brain volume, smaller ventricular volume, larger gangliothalamic ovoid diameters and cortical thickness (10-13).

Recent advances in imaging technology have made it possible to noninvasively evaluate the effects of infant diets on brain structure/morphology underlying these observations with greater precision than ever before (14). One aspect of brain structure where diet-related differences might be expected would be in the integrity of white matter. White matter contains large bundles of myelinated axons and is responsible for transmission of signals between different brain regions, and breast milk and formulas differ in factors that contribute to myelination (1). The structural connectivity provided by white matter tracts is essential for normal brain function, and abnormalities or deficits in white matter have been observed in a wide variety of neurodevelopmental disorders in children (15-18). In addition, positive correlations between white matter integrity and brain functioning such as IQ, memory, and reading in healthy children have been reported (19-21). Therefore, utilizing advanced neuroimaging methods to measure the comparative effects of breastfeeding or formula feeding on brain white matter development could reveal potential mechanisms underlying the well-known benefits of breastfeeding.

Diffusion tensor imaging (DTI) is a magnetic resonance imaging (MRI) method that can provide noninvasive visualization of white matter tracts by tractography, and the DTI parameter fractional anisotropy (FA) is very sensitive to white matter integrity. As a measure of water molecule diffusion

${ }^{1}$ Arkansas Children's Nutrition Center, Little Rock, Arkansas; ${ }^{2}$ Department of Pediatrics, University of Arkansas for Medical Sciences, Little Rock, Arkansas; ${ }^{3}$ Department of Radiology, University of Arkansas for Medical Sciences, Little Rock, Arkansas; ${ }^{4}$ Vanderbilt University Institute of Imaging Sciences, Nashville, Tennessee. Correspondence: Xiawei Ou (OuXiawei@uams.edu) 
anisotropy, FA values are high in myelinated white matter because the myelin sheath resists water diffusion perpendicular to white matter tracts while axonal bundles facilitate water diffusion parallel to white matter tracts. FA measurements have been used to identify white matter pathology not evident on conventional MRI $(22,23)$. Furthermore, a recently developed DTI analysis method, tract-based spatial analysis (TBSS), can provide non-subjective voxel-wise analysis of FA values for the whole brain (24), and has shown great sensitivity to white matter abnormalities $(25,26)$.

While two recent papers reported effects of breast milk on different aspects of white matter (i.e., total white matter volume, myelin-associated water pool fraction) in different pediatric populations $(12,27)$, characterizing early diet effects on white matter by DTI has not been previously reported. Furthermore, sex differences were found in white matter growth profiles (28), but sex-specific evaluation of diet effects on healthy children (term-born) at school age has not been studied. In this study, we used DTI TBSS to evaluate brain white matter integrity in healthy 8 -y-old children who were predominately BF or FF as infants. We correlated DTI measured FA with IQ and language test scores. We also used both TBSS and ROI methods to further compare the white matter in subsets of children with exclusive breast milk feeding or formula feeding for at least the first year of life. Based on reports associating breastfeeding with better neurodevelopment, we hypothesized that breastfeeding would be associated with greater white matter integrity than formula feeding, and these effects would be sex specific.

\section{RESULTS}

Fifty-six children completed this study, including 22 predominantly BF and 34 predominantly FF as infants. Predominantly $\mathrm{BF}$ infants were breastfed for at least 6 mo before transitioning to a milk-based formula, but most were breastfed for at least 8 mo. The mean and standard deviation of breastfeeding duration for the BF infants were $12.5 \pm 6.2$ mo. Predominantly FF infants were fed formula for at least $8 \mathrm{mo}$. Infants were introduced to complimentary foods on average around 5 mo of age. Among these participants, 10 children were exclusively breastfed (EBF) from birth through age 12 mo (breastfeeding duration $16.2 \pm 7.6 \mathrm{mo}$ ), and 13 were exclusively formulafed (EFF) between ages $1 \mathrm{wk}$ to 12 mo. "Exclusively" refers to infants who started on either breastfeeding starting at birth or formula feeding starting by age $1 \mathrm{wk}$ and remained on the same food source until complimentary foods were introduced and through weaning. The $n$ /group, ages at MRI, and other demographic information are listed in Table 1 . There was no significant difference in age at MRI, race distribution, or sex distribution between feeding groups. In addition, there was no significant difference in the age distribution of girls and boys by feeding group.

There were no significant group differences in whole brain FA values between BF and FF children $(0.394 \pm 0.011$ vs. $0.393 \pm 0.014, P=0.71)$, or between EBF and EFF children $(0.398 \pm 0.011$ vs. $0.390 \pm 0.017, P=0.20)$. However,
Table 1. Demographic information of the subjects

\begin{tabular}{lccc}
\hline All subjects & BF $(N=22)$ & FF $(N=34)$ & Pvalue \\
\hline $\begin{array}{l}\text { Age at MRI } \\
\text { (mean } \pm \text { SD) }\end{array}$ & $7.98 \pm 0.29$ & $7.89 \pm 0.23$ & 0.24 \\
Race & & & \\
Caucasian (\%) & $20(90.9)$ & $28(82.4)$ & \\
African American (\%) & $1(4.5)$ & $4(11.8)$ & 0.838 \\
Other (\%) & $1(4.5)$ & $2(5.9)$ & \\
Child's sex & & & 0.999 \\
Girls (\%) & $12(54.5)$ & $18(52.9)$ & 0.975 \\
Boys (\%) & $10(45.5)$ & $16(47.1)$ & \\
\hline Exclusive feeding & EBF (N=10) & EFF (N=13) & \\
\hline Age at MRI & & \\
(mean \pm SD) & $7.88 \pm 0.37$ & $7.86 \pm 0.25$ & \\
Race & & & 0.999 \\
Caucasian (\%) & $9(90.0)$ & $8(61.5)$ & \\
African American (\%) & $1(10.0)$ & $4(30.8)$ & \\
Other (\%) & $0(0.0)$ & $1(7.7)$ & \\
Child's sex & & & \\
Girls (\%) & $4(40.0)$ & $6(46.2)$ & \\
Boys (\%) & $6(60.0)$ & $7(53.8)$ & \\
\hline BF, & & & \\
\hline
\end{tabular}

$B F$, breastfed; E, exclusive; $F F$, formula-fed; $M R I$, magnetic resonance imaging.

there were sex-specific effects. Whole brain mean FA values were significantly higher in $\mathrm{BF}$ than FF males $(0.398 \pm 0.011$ vs. $0.390 \pm 0.009, P=0.04)$ and in $\mathrm{EBF}$ than EFF males (0.401 \pm 0.007 vs. $0.386 \pm 0.007, P=0.008)$, but did not differ in BF and FF females $(0.392 \pm 0.011$ vs. $0.395 \pm 0.017, P=0.32)$ or in EBF and EFF females $(0.393 \pm 0.015$ vs. $0.393 \pm 0.024$, $P=0.87)$.

When all children in the study were considered together, no significant diet group (BF vs. FF) or sex differences were noted in regional FA values by the voxel-wise TBSS analysis. However, within sex between-group analyses showed significantly higher FA values $(P<0.05$, corrected) in BF compared to FF males in multiple white matter regions of the left hemisphere (Figure 1). These regions generally corresponded to the superior longitudinal fasciculus, cingulum, body of corpus callosum, and posterior crossing fibers (crossing of forceps major and posterior thalamic radiation). No region had significantly lower FA values in BF than FF males, and females in BF and FF groups did not show significant differences in FA values.

When we narrowed the TBSS analyses to just those children in the EBF or EFF groups, we found widespread regions with higher FA values $(P<0.05$, uncorrected) in EBF than EFF children (Figure 2). No regions with lower FA values were noted. When the data were corrected for multiple comparisons, sexspecific analysis revealed extensive regions of white matter with significantly higher FA values $(P<0.05$, corrected $)$ in EBF compared to EFF males (Figure 3 ). These regions were also located in the left hemisphere and included the superior 


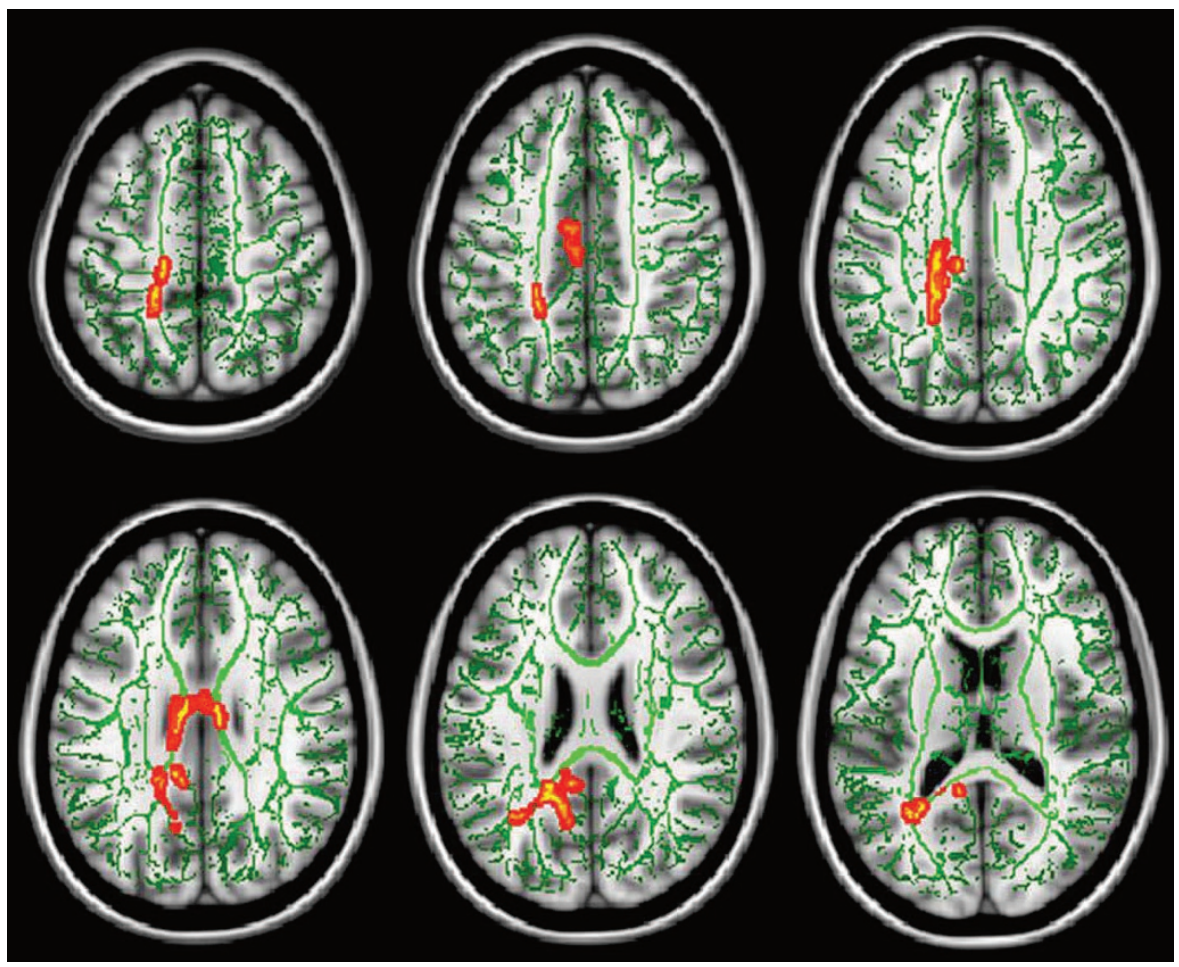

Figure 1. White matter differences between breastfed (BF) and formula-fed (FF) males. Mean fractional anisotropy (FA) skeleton (green) were overlaid on T1-weighted magnetic resonance imaging at six different positions (different images in the figure) of the MNI152 brain template. Orange/yellow represents white matter regions with significantly higher FA values $(P<0.05$, corrected) in BF than FF males.

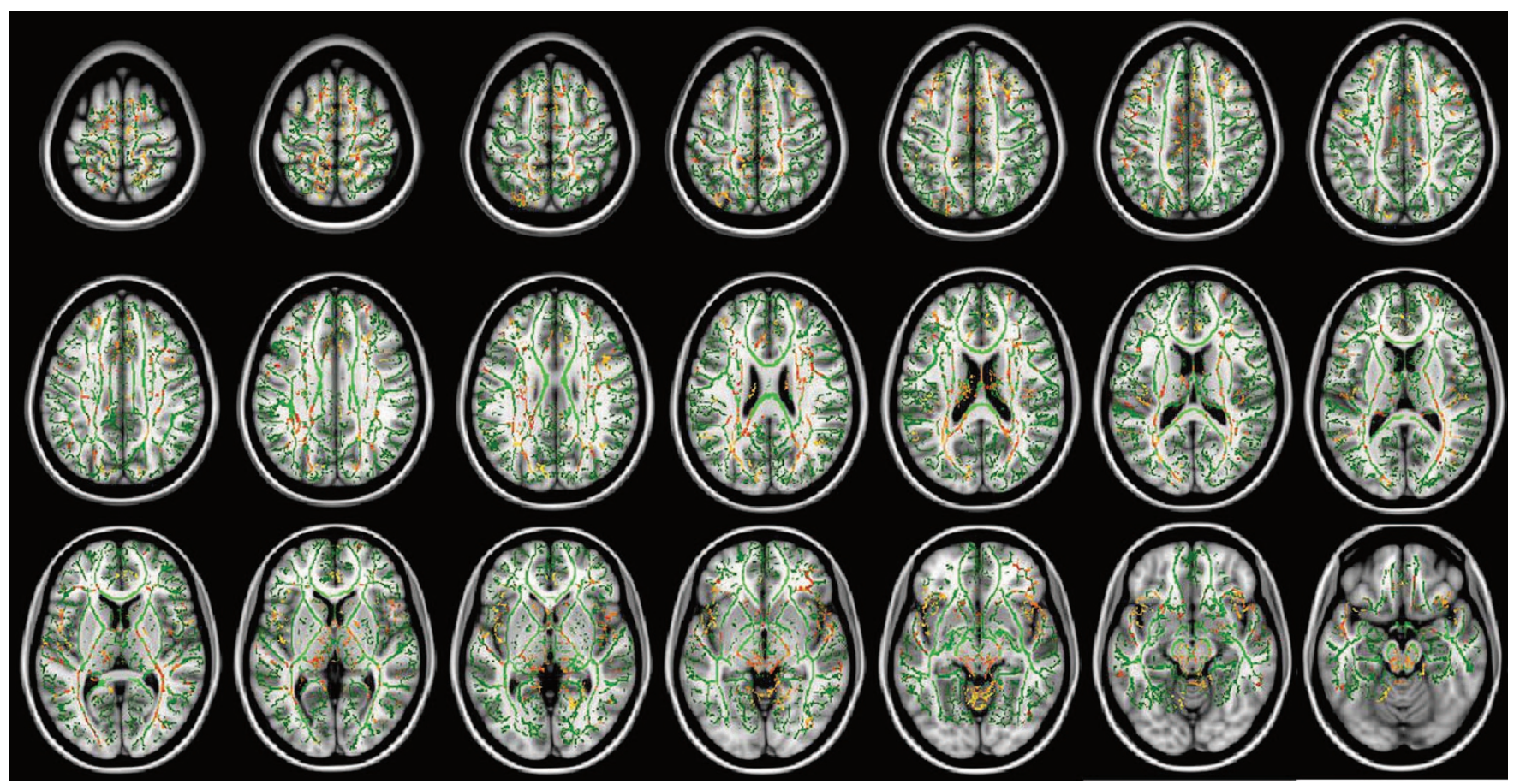

Figure 2. White matter differences between exclusive breastfed (EBF) and exclusive formula-fed (EFF) groups (before correction for multiple comparisons). Mean fractional anisotropy (FA) skeleton (green) were overlaid on T1-weighted magnetic resonance imaging at 21 different positions (different images in the figure) of the MNI152 brain template. Orange/yellow represents white matter pixels with higher FA values $(P<0.05$, uncorrected) in EBF than EFF groups.

longitudinal fasciculus, superior and frontal corona radiata, external capsule, posterior limb of interior capsule, and the posterior crossing fibers (crossing of forceps major and posterior thalamic radiation). No region had significantly lower FA in males and there were no significant differences in FA values in EBF vs. EFF females. 


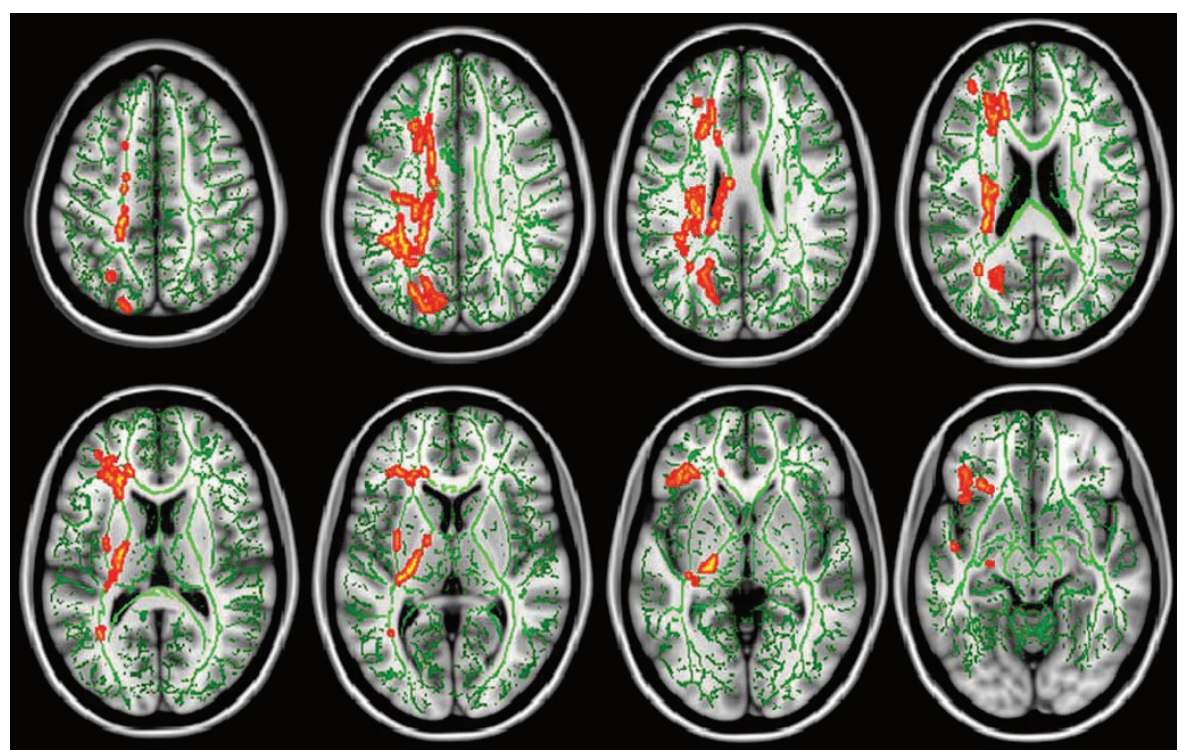

Figure 3. White matter differences between exclusive breastfed (EBF) and exclusive formula-fed (EFF) males. Mean fractional anisotropy (FA) skeleton (green) were overlaid on T1-weighted magnetic resonance imaging at 8 different positions (different images in the figure) of the MNI152 brain template. Orange/yellow represents white matter regions with significantly higher FA values $(P<0.05$, corrected) in EBF males than EFF males.

We further performed ROI analysis to compare the FA values in the EBF and EFF children based on (i) the indicative TBSS comparison (before correction) of these subgroups in Figure 2; and (ii) the benefit of increasing the statistical power by decreasing number of comparisons (from voxelwise to region-wise) in lieu of the relatively smaller sample size of the EBF and EFF subgroups. Eleven clusters were identified with greater FA values in EBF than EFF children with a less stringent statistical threshold $(P<0.15$ after multiple comparisons correction by TBSS with TFCE) and a cluster size threshold of $>14$ (Table 2). These clusters were located in nine white matter regions which were subsequently selected for the ROI analysis and included the cerebral peduncles; the right optic radiation; the left posterior crossing fibers (the crossing of forceps major and posterior thalamic radiation); the right superior longitudinal fasciculus; the right posterior limb of internal capsule; the right posterior crossing fibers; the right superior frontal white matter; the right superior parietal white matter; and the left parietal occipital white matter. The mean FA values and standard deviation for the EBF and EFF subgroups are listed in Table 3. When stratified by sex, females did not show any region with significant FA differences between EBF and EFF groups, while males showed significantly higher FA in EBF than EFF in all but one of the 9 regions.

None of the neuropsychological test parameters (the Reynolds Intellectual Assessment Scale (RIAS)) and language skills (Clinical Evaluation of Language Fundamentals (CELF4)) differed significantly between BF and FF groups (Table 4). There were no significant sex related effects. Most of the neuropsychological tests, except the Nonverbal intelligence index (NIX), showed a trend of higher scores in EBF than EFF groups (Table 4), however, the difference was only significant in the expressive language (EXP) scores $(107 \pm 13$ vs. $95 \pm 12$
Table 2. Brain white matter clusters as regions of interest for the exclusive feeding subgroups

\begin{tabular}{|c|c|c|c|c|c|}
\hline \multirow{2}{*}{$\begin{array}{l}\text { Corrected } \\
P \text { value }\end{array}$} & \multirow{2}{*}{$\begin{array}{l}\text { Cluster } \\
\text { size }\left(\mathrm{mm}^{3}\right)\end{array}$} & \multicolumn{3}{|c|}{$\begin{array}{l}\text { MNI152 coordinates } \\
\text { (center of cluster) }\end{array}$} & \multirow[b]{2}{*}{ Location } \\
\hline & & $x$ & $y$ & $z$ & \\
\hline 0.082 & 1,249 & -27 & -51 & 21 & $\begin{array}{l}\text { Left posterior crossing } \\
\text { fibers }\end{array}$ \\
\hline 0.104 & 1,002 & 19 & -46 & 51 & $\begin{array}{l}\text { Right superior } \\
\text { longitudinal fasciculus }\end{array}$ \\
\hline 0.104 & 2,218 & -4 & -25 & -5 & Cerebral peduncle \\
\hline 0.112 & 750 & 22 & -8 & 15 & $\begin{array}{l}\text { Right posterior limb of } \\
\text { internal capsule }\end{array}$ \\
\hline 0.118 & 1,350 & 32 & -52 & 14 & Right optic radiation \\
\hline 0.14 & 101 & 30 & 16 & 52 & $\begin{array}{l}\text { Right superior frontal } \\
\text { white matter }\end{array}$ \\
\hline 0.148 & 138 & 20 & -51 & 22 & $\begin{array}{l}\text { Right posterior crossing } \\
\text { fibers }\end{array}$ \\
\hline 0.148 & 36 & 18 & -15 & -5 & $\begin{array}{l}\text { Right cerebral peduncle/ } \\
\text { internal capsule }\end{array}$ \\
\hline 0.15 & 18 & 16 & -18 & -11 & Right cerebral peduncle \\
\hline 0.15 & 14 & 25 & -43 & 68 & $\begin{array}{l}\text { Right superior parietal } \\
\text { white matter }\end{array}$ \\
\hline 0.15 & 14 & -16 & -79 & 36 & $\begin{array}{l}\text { Left parietal occipital } \\
\text { white matter }\end{array}$ \\
\hline
\end{tabular}

All clusters with size $\geq 14$ voxels and $P$ values (after multiple comparison corrections) $\leq 0.15$ are listed.

$P=0.03)$. Except for the RIAS NIX, all test scores showed significant positive correlations with whole brain mean FA values (Figure 4) when consider all BF and FF children. For the exclusive feeding subgroups (EBF and EFF) which had a smaller sample size, the correlation was only significant between the verbal intelligence index (VIX) and whole brain mean FA values $(r=0.46, P=0.03)$. 
Table 3. Sex-specific comparisons of fractional anisotropy values for the exclusive feeding subgroups

\begin{tabular}{|c|c|c|c|c|c|c|}
\hline & \multicolumn{3}{|c|}{ Females } & \multicolumn{3}{|c|}{ Males } \\
\hline & EBF & EFF & $P$ value & EBF & EFF & $P$ value \\
\hline WBWM & $0.393 \pm 0.015$ & $0.393 \pm 0.024$ & 0.870 & $0.401 \pm 0.007$ & $0.386 \pm 0.007$ & 0.008 \\
\hline ROR & $0.650 \pm 0.040$ & $0.623 \pm 0.041$ & 0.476 & $0.647 \pm 0.026$ & $0.592 \pm 0.025$ & $0.005^{*}$ \\
\hline LPCR & $0.574 \pm 0.026$ & $0.536 \pm 0.059$ & 0.257 & $0.628 \pm 0.046$ & $0.528 \pm 0.072$ & 0.035 \\
\hline RPLIC & $0.703 \pm 0.026$ & $0.701 \pm 0.025$ & 0.914 & $0.713 \pm 0.028$ & $0.677 \pm 0.018$ & 0.035 \\
\hline R PCR & $0.570 \pm 0.082$ & $0.499 \pm 0.051$ & 0.171 & $0.574 \pm 0.087$ & $0.510 \pm 0.065$ & 0.138 \\
\hline R SFW & $0.438 \pm 0.025$ & $0.389 \pm 0.050$ & 0.114 & $0.482 \pm 0.025$ & $0.371 \pm 0.019$ & $0.001 *$ \\
\hline R SPW & $0.268 \pm 0.027$ & $0.253 \pm 0.019$ & 0.610 & $0.306 \pm 0.060$ & $0.243 \pm 0.028$ & 0.035 \\
\hline LPOW & $0.338 \pm 0.029$ & $0.347 \pm 0.033$ & 0.914 & $0.374 \pm 0.043$ & $0.325 \pm 0.022$ & 0.022 \\
\hline
\end{tabular}

CP, cerebral peduncles; EBF, exclusive breastfed; EFF, exclusive formula-fed; L, left; OR, optic radiation; PCR, posterior crossing fibers; PLIC, posterior limb of internal capsule; POW, parietal occipital white matter; R, right; SFW, superior frontal white matter; SLF, superior longitudinal fasciculus; SPW, superior parietal white matter; WBWM, whole brain white matter. $P$ values in bold reflect significant differences between EBF and EFF.

${ }^{*} P<0.05$ after Bonferroni correction.

Table 4. RIAS and CELF-4 test scores

\begin{tabular}{|c|c|c|c|c|c|c|c|}
\hline & & $\mathrm{BF}$ & $\mathrm{FF}$ & $P$ value & EBF & EFF & $P$ value \\
\hline \multicolumn{8}{|l|}{ RIAS } \\
\hline \multirow[t]{3}{*}{ Verbal IQ (VIX) } & All & $109 \pm 16$ & $107 \pm 14$ & 0.37 & $112 \pm 18$ & $100 \pm 9$ & 0.14 \\
\hline & Female & $110 \pm 11$ & $107 \pm 11$ & 0.36 & $111 \pm 16$ & $103 \pm 8$ & 0.72 \\
\hline & Male & $109 \pm 21$ & $107 \pm 18$ & 0.82 & $113 \pm 21$ & $97 \pm 11$ & 0.19 \\
\hline \multirow[t]{3}{*}{ Non-verbal IQ (NIX) } & All & $111 \pm 13$ & $116 \pm 15$ & 0.37 & $110 \pm 14$ & $113 \pm 13$ & 0.95 \\
\hline & Female & $115 \pm 11$ & $116 \pm 13$ & 0.6 & $118 \pm 9$ & $114 \pm 13$ & 0.41 \\
\hline & Male & $106 \pm 14$ & $116 \pm 18$ & 0.16 & $105 \pm 16$ & $112 \pm 15$ & 0.61 \\
\hline \multirow[t]{3}{*}{ Composite IQ (CIX) } & All & $111 \pm 13$ & $111 \pm 13$ & 0.99 & $111 \pm 16$ & $106 \pm 10$ & 0.37 \\
\hline & Female & $113 \pm 9$ & $112 \pm 11$ & 0.54 & $114 \pm 10$ & $108 \pm 11$ & 0.25 \\
\hline & Male & $107 \pm 17$ & $112 \pm 16$ & 0.56 & $109 \pm 19$ & $103 \pm 10$ & 0.56 \\
\hline \multicolumn{8}{|l|}{ CELF-4 } \\
\hline \multirow[t]{3}{*}{ Receptive language (REC) } & All & $108 \pm 10$ & $106 \pm 11$ & 0.61 & $110 \pm 12$ & $102 \pm 11$ & 0.19 \\
\hline & Female & $109 \pm 6$ & $108 \pm 10$ & 0.98 & $112 \pm 3$ & $105 \pm 8$ & 0.24 \\
\hline & Male & $107 \pm 14$ & $104 \pm 12$ & 0.78 & $109 \pm 15$ & $99 \pm 14$ & 0.51 \\
\hline \multirow[t]{3}{*}{ Expressive language (EXP) } & All & $104 \pm 11$ & $102 \pm 15$ & 0.58 & $107 \pm 13$ & $95 \pm 12$ & 0.03 \\
\hline & Female & $107 \pm 10$ & $101 \pm 15$ & 0.3 & $110 \pm 13$ & $97 \pm 9$ & 0.06 \\
\hline & Male & $101 \pm 13$ & $102 \pm 16$ & 0.8 & $105 \pm 13$ & $93 \pm 15$ & 0.25 \\
\hline \multirow[t]{3}{*}{ Core language (CORE) } & All & $104 \pm 12$ & $102 \pm 14$ & 0.63 & $107 \pm 14$ & $95 \pm 12$ & 0.06 \\
\hline & Female & $107 \pm 9$ & $103 \pm 14$ & 0.47 & $110 \pm 12$ & $97 \pm 8$ & 0.07 \\
\hline & Male & $102 \pm 15$ & $102 \pm 16$ & 0.82 & $104 \pm 16$ & $93 \pm 16$ & 0.32 \\
\hline
\end{tabular}

BF, breastfed; CELF-4, Clinical Evaluation of Language Fundamentals; FF, formula-fed; RIAS, Reynolds Intellectual Assessment Scale.

\section{DISCUSSION}

This study is the first to relate differences in early diet with later brain development using DTI measures. Our results show that 8-y-old male children who were BF as infants had significantly higher FA values than FF males in multiple white matter regions. These regions are limited to the left hemisphere and included the body of the corpus callosum which facilitates inter-hemisphere communication, part of the posterior superior longitudinal fasciculus which may be involved in language (29), and part of the posterior cingulum which may be involved in working and general memory (30). When considering only children who were exclusively breastfed or formula-fed for the first year of life, EBF males showed more widespread regions with higher FA values than EFF males in the left hemisphere, including the majority of the superior longitudinal fasciculus and frontal white matter, which may be 


\section{Articles | ouetal.}
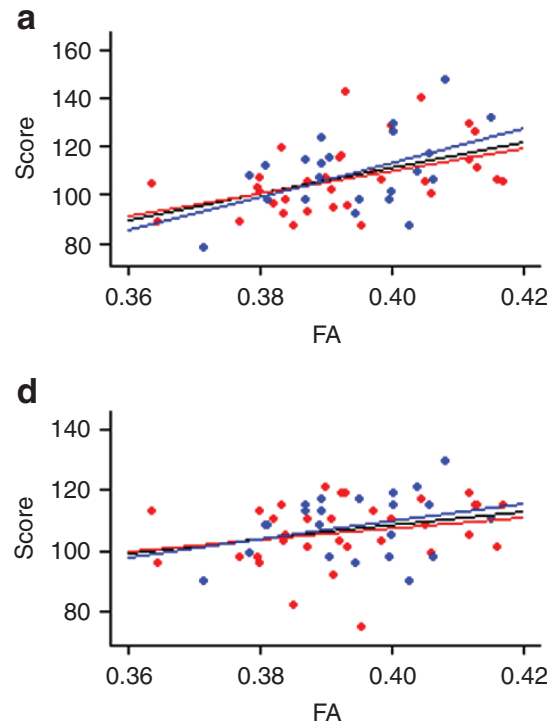

b

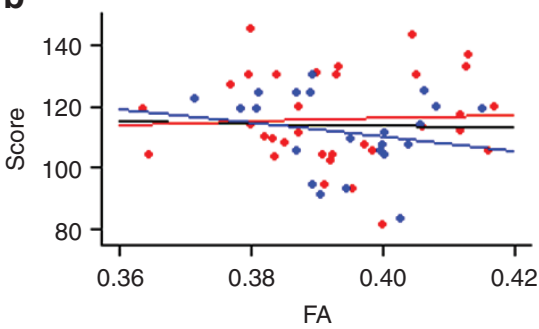

e

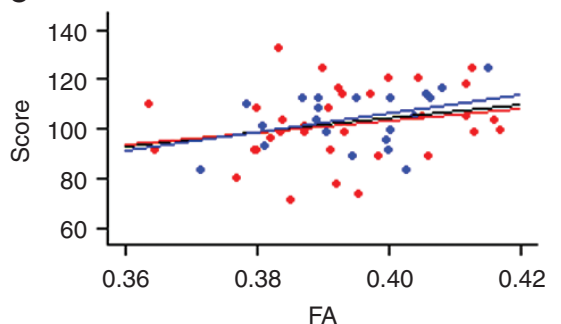

C

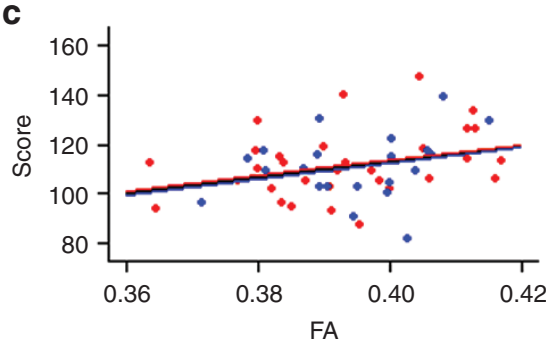

f

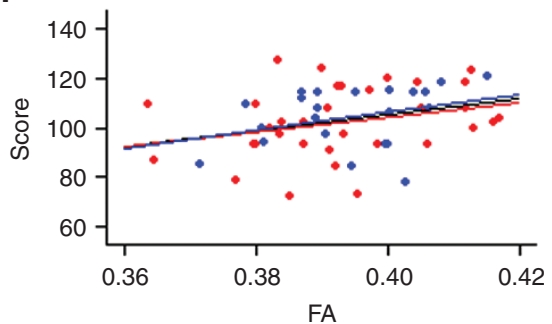

Figure 4. Correlations of test scores with mean fractional anisotropy (FA) values. All RIAS and CELF-4 scores (except for Nonverbal intelligence index (NIX)) showed significant positive correlations with whole brain white matter mean FA values: $(\mathbf{a}) \mathrm{VIX}: r=0.45, P<0.01 ;(\mathbf{b}) \mathrm{NIX}: r=-0.03, P=0.82$; (c) CIX: $r=0.30, P=0.02$; (d) REC: $r=0.29, P=0.03$; (e) EXP: $r=0.28, P=0.04$; (f) CORE: $r=0.30, P=0.03$. Blue and red dots represent breastfed (BF) and formula-fed (FF) subjects, respectively; black, blue, and red lines are the least-squares fit lines for all, BF, and FF subjects, respectively.

important for language and cognitive functions (31), and the posterior limb of internal capsule which may be related with the motor functioning (32). In addition to the non-subjective voxel-wise evaluation of white matter by TBSS, a more subjective region of interest analysis revealed FA differences in additional regions (including some in the right hemisphere) between EBF and EFF males. Moreover, whole brain white matter mean FA values were significantly higher in BF than FF males and in EBF than EFF males. These findings suggest that boys with predominant breast milk feeding as infants have overall better white matter integrity, with the differences more prominent in brain regions associated with language/memory functions and in children with exclusive and prolonged breast milk feeding for at least $1 \mathrm{y}$.

These diet-related white matter effects were not observed in 8 -y-old girls. While it is possible that there are subtle effects in females which cannot be detected with our sample size, our data suggest that the effects of breast milk on brain white matter integrity are sex specific. Sex differences in white matter integrity as a function of infant diet have not been reported in young children who were term-born. However, sex differences in developmental trajectories of white matter in healthy adolescents, as well as sex differences of white matter in healthy adults, have been previously reported by DTI studies $(28,33)$. Furthermore, a number of studies have shown that effects of infant diet on later growth and development are more prominent in male children. For example, Oddy et al. (7) found that benefits of breastfeeding on academic achievement were significant in boys but not girls, and speculated that because of the relatively lower concentrations of neuroprotective estrogens in boys, they derived greater benefits from neuroprotective breast milk. Similarly, in a large randomized controlled trial, Kramer et al. (6) found slightly higher positive effects of breastfeeding on verbal IQ in boys than girls. In addition, Nagahara et al. (34) observed significant effects of breastfeeding vs. formula feeding on the growth of healthy boys, but no significant differences in girls. Finally, in a randomized controlled trial comparing preterm formula vs. standard formula, significant effects on verbal IQ at 7.5-8 y were observed in boys, but not girls (35).

The DTI measured FA values in our study correlated significantly with verbal IQ and composite IQ from the RIAS, and receptive, expressive, and core language scores from the CELF4. The correlation of FA with IQ is consistent with previous DTI findings in normal children (21). The correlation of FA with language test scores has also been reported in normal children (20), and in children born preterm (36). Our findings of significant correlation between FA and verbal IQ/language scores are also consistent with the TBSS findings of white matter regions involved in language functioning (e.g., the superior longitudinal fasciculus and the left frontal gyrus) and suggest that language development may be an important outcome to assess in studies of infant diet effects. While a number of studies with larger sample sizes have shown significant effects of breastfeeding on IQ in school age children $(2,35)$, the RIAS IQ and CELF-4 language scores measured in our study were not significantly different between 8-y-old term-born BF or FF children. However, significant group differences in white matter regional FA values associated with language were present. These results suggest that subtle differences in specific brain white matter regions may exist even though outcome measures do not yet show differences and warrant further investigation. For EBF children who had exclusive and prolonged $(>12 \mathrm{mo})$ breastfeeding, the expressive language scores were significantly higher than that in EFF children, consistent with the more prominent brain differences as reflected by more widespread regions with higher FA values. 
We are aware of two other studies addressing the effects of infant diet on brain white matter integrity later in life. Isaacs et al. (12) measured brain volume in adolescents (13-19 y of age) who were born preterm ( $\leq 30 \mathrm{wk}$ gestation age) but were neurologically normal in childhood. These infants were randomized to one of a series of neonatal diets, and a positive correlation was found between the percentage of total calories consumed as expressed breast milk and total white matter volume in boys, but not in girls. Those findings of sex-specific effects of breast milk on white matter are consistent with the results reported herein. In a cross-sectional study of children aged 1-4 y in which mothers self-reported year one infant diet intake, Deoni et al. (27) measured myelin-associated water pool fraction in white matter in children fed by exclusive breastfeeding, exclusive infant formula-feeding, or a combination of breastfeeding and formula-feeding. While sex-specific results were not reported, increased white matter integrity was found in later maturing frontal and association regions in all children with exclusive breastfeeding. Similarly, TBSS and ROI analyses on EBF and EFF children in this study identified multiple frontal and association white matter regions which had better white matter integrity associated with exclusive breastfeeding. Furthermore, we found significant correlations of white matter FA values with verbal IQ and CELF language scores, which is consistent with findings of Isaacs et al. showing a possible correlation between white matter volume and verbal IQ. Neither our results nor those of Isaacs et al. found effects on nonverbal performance IQ.

The mechanism(s) underlying greater white matter integrity in breastfed children is yet to be determined. One speculation is that breast milk promotes the myelination process, which happens mostly in the first year of life. Breast milk is a complex mixture of compounds, many of which (e.g., omega-3 fatty acids, sialic acid, and choline) are thought to be important in myelination, cell structure, and function. In addition, breast milk contains human hormones, growth factors and maternal cells, all of which are either absent in infant formulas or present at much lower levels (1). Another possible mechanism by which breast milk might influence brain myelination is via its effects on the development of brain electroencephalographic (EEG) activity during infancy. Studies have shown that brain electrical activity may promote myelination $(37,38)$ and the timing and extent of increases in brain neural activity may differentially affect the myelination process. During infancy EEG development follows a different time course in $\mathrm{BF}$ and FF infants (39). Furthermore, infant diet-related differences in surges in EEG activity in the 9-30 Hz frequency range were greatest among boys, with $\mathrm{BF}$ boys showing significantly greater activity than FF boys (39), a finding consistent with the greater FA values in BF than FF males in this study. Animal studies have also revealed a tendency of decreased $21.5-\mathrm{kDa}$ isoform of myelin basic protein in the prefrontal cortex in early weaned neonatal rats (40), where less breast milk intake occurs and which may affect the myelin formation pattern. However, direct quantification of white matter myelination is difficult in vivo. While the myelin-associated water pool fraction measured by mcDESPOT MRI pulse sequence reported by others (27) may detect white matter microstructural differences in children and may be myelin specific, it is yet to be determined whether this parameter is a sole reflection of myelin. On the other hand, DTI is a well-established imaging method for white matter quantification and the great sensitivity of FA to myelination is well recognized. Therefore, DTI results in this study provide important new data in understanding effects of breastfeeding to white matter development.

Normal CNS development is thought to best be achieved with a full term gestation. During gestation, nutrients and bioactive factors such as hormones and growth factors provided by the intra-uterine environment play essential roles in this development. However, rapid CNS development continues during the first 12 postnatal months of life, and even small nutrient deficiencies during this time could prevent children from attaining their individual optimal brain development. Relative to breastfeeding, infant formulas could very well represent a nutrient and/or growth factor deficient food with respect to promoting optimal brain development/maturation. To the extent that DTI-measured FA is a reflection of white matter integrity, including myelination which represents a structural brain component required for CNS function (i.e., neural transmission), it is not surprising that multiple studies have demonstrated better neurocognitive development, higher scores on standardized behavioral tests, higher intelligence quotient (IQ), and better school performance in BF children.

This study is not without limitations. While we studied the effects of exclusive and prolonged breast milk feeding $(\geq 12$ mo), the sample sizes for these subgroups were relatively small. We incorporated ROI analysis to investigate whether exclusive breast milk feeding for at least the first year of life would benefit the white matter integrity in both males and females, however, significant improvement was still only observed in males, though a trend of higher FA was shown in several regions for females. Nevertheless, ROI analysis is subjective because the morphological definition of many white matter regions is not distinct and identification of the regions requires observer judgment which inevitably results in statistical variability. Another limitation is the lack of background information regarding home environment variables such as socioeconomic status, family income, parental education, mom's IQ, and stress. While our recruiting method might be expected to attenuate group differences on these variables, it remains possible that some of these factors interacted with the diet effects we found in this study.

In conclusion, our results based on DTI FA measurements indicate better white matter integrity in breastfed than formula-fed boys. Furthermore, FA measurements correlated with RIAS verbal IQ and CELF-4 language scores in 8-yold healthy children. These results reveal two potentially important concepts relative to infant diets: (i) they suggest that what a child is fed immediately following a normal term birth can influence brain development, and potentially have long-term (perhaps permanent) effects on brain structure and function, that are evident in preadolescents, at least 
until age 8 years; (ii) breastfeeding may differentially influence white matter integrity in males compared to females, suggesting that boys may benefit more from exclusive breastfeeding than girls.

\section{METHODS \\ Subjects}

Healthy children (age 7.5-8.5 y) with parental reports of predominant breast milk or infant formula feeding from birth through age $12 \mathrm{mo}$ were recruited from the local population. Participants were recruited from the central Arkansas region via advertisements at health fairs, pediatrician offices, summer camps, schools, social media and community events. Prior to participation, informed consent was obtained from parents and assent from children. The study protocol was approved by the Institutional Review Board of the University of Arkansas for Medical Sciences and is part of ClinicalTrials.gov NCT00735423. All children were born full-term with birth weight between 5th and 95th percentile-for-age. Children of mothers with alcohol, tobacco, drug, or psychotropic medication use during pregnancy and children with psychological/psychiatric diagnoses or neurological impairment or injury were excluded.

\section{Study Visits}

There were three planned study visits. During the first visit, assessments were made to determine eligibility for the study. Growth parameters such as height, body weight, and body mass index were also measured at this time. MRI scanning with DTI was performed at the Arkansas Children's Hospital on the second visit. If the participants successfully completed the MRI scan, they were administered standardized tests of intelligence (the RIAS and CELF-4) during a third visit.

MRI examination. MRI scans were performed on a 1.5 Tesla Achieva MRI scanner (Philips Healthcare, Best, Netherlands). The built-in body coil was used as the transmitter and a standard 8-channel SENSE head coil was used as the receiver. A single-shot spin echo EPI sequence with diffusion weighting gradients in 15 uniformly distributed directions was used to acquire DTI data. Parameters for the DTI sequence included $5135 \mathrm{~ms}$ TR, $66 \mathrm{~ms}$ TE, $220 \times 220 \mathrm{~mm}$ field of view, $2 \times 2 \times$ $3 \mathrm{~mm}$ voxel size, $128 \times 128$ reconstruction matrix size, 34 contiguous axial slices, 2 averages, and a maximum b value was $800 \mathrm{~s} / \mathrm{mm}^{2}$.

Neuropsychological tests. The RIAS consisted of two subtests for Verbal Intelligence Index (measuring vocabulary knowledge and analytical reasoning abilities), two subtests providing a Nonverbal Intelligence Index (measuring general reasoning skills emphasizing nonverbal ability, and nonverbal reasoning skills through the presentation of pictures), and a Composite Index which reflects overall intelligence and reasoning. The CELF-4 test included six language subtests: Concepts and Following Directions; Word Structure; Recalling Sentences; Sentence Structure; Formulated Sentences; and Word Classes Receptive. The scaled scores for the subtests were combined into a receptive language score, an expressive language score, and a core language score which reflects the overall language abilities. Licensed psychological examiners under the supervision of certified clinical psychologists conducted all testing.

\section{Data Analysis}

DTI TBSS analysis. Fractional anisotropy maps for each subject were calculated on the scanner from the diffusion tensor images by Fibertrak (Philips Healthcare, Best, Netherlands). FA maps were then exported to a workstation with MATLAB software (The Mathworks, Natick, MA) and FMRIB Software Library (FSL, Oxford, UK) installed on a VMware Linux virtue machine (VMware, Palo Alto, CA). Voxelwise analysis of the FA data was carried out using TBSS (24) in FSL. Each FA data set was first pre-processed to remove outliers and zero end slices before nonlinear registration. Then, every FA data set was aligned to every other one to identify the most representative set which has the smallest amount of average warping and this FA set was consequently served as the target. The target FA data set was then affine-aligned into the Montreal Neurological Institute standard brain (MNI152 space), and every FA data set was then transformed into this
$1 \times 1 \times 1 \mathrm{~mm}$ MNI152 space by combining nonlinear registration to the target and affine transform from the target to the MNI152 space. All FA images were merged, averaged, and fed into the FA skeletonization program to create a mean FA skeleton in which a threshold of FA $\geq 0.2$ was chosen. The alignment of the FA data and the formation of the FA skeleton were carefully reviewed before proceeding to voxel-wise group and sex statistical comparisons. The whole brain white matter skeleton mean FA value for each participant was also calculated from the TBSS analysis.

Neuropsychological tests and correlation with DTI. Outcome scores from the RIAS (VIX, NIX, and CIX) and CELF-4 (REC, EXP, and CORE) were compared between groups, between boys and girls within group, and within sex across groups. In addition, these measures were correlated with mean FA values in whole brain white matter.

Subgroup data analysis. EBF and EFF: Separated analyses were conducted comparing subsets of the total sample (EBF and EFF) that had exclusive and prolonged breast milk or formula feeding. Neuropsychological and DTI TBSS subgroup analyses followed procedures described above and included similar sex-specific group comparisons. In addition, FA skeleton maps were used for ROI analysis of FA values. The ROI analysis was performed on the normalized axial FA skeleton maps instead of the original FA maps to limit intersubject variation in the region selection and to increase the sensitivity because only the center of the white matter tracts (the skeleton) was considered. White matter regions (as defined by anatomy) which contained clusters with FA differences (at a statistical threshold specified in the Results section) between all EBF and all EFF children in the TBSS analysis were chosen as the ROIs. The mean FA value in each ROI was calculated and compared between groups.

\section{Statistical Analysis}

Fisher's exact test was used to compare categorical demographic characteristics across feeding groups, whereas the Wilcoxon Rank-Sum test was used to compare continuous demographic characteristics, RIAS and CELF-4 scores, and DTI ROI based FA values across feeding groups. Statistical significance was set at $P=0.05$. For correlations between RIAS/CELF-4 scores and mean FA values, a test for normality was first performed. If $P<0.05$, the Spearman Correlation test was used; whereas, if $P>0.05$, either the Pearson or the Spearman Correlation test was used, whichever had the smaller $P$ value. For the voxel-wise analysis by TBSS, randomization with 3,000 permutations was used with Threshold-Free Cluster Enhancement (TFCE) to detect voxels with significant FA values between groups $(P<0.05)$ after family-wise error (FWE) correction for multiple comparisons.

\section{ACKNOWLEDGMENTS}

We appreciate the efforts of the Arkansas Children's Nutrition Center's Human Studies Core for all their efforts in this project. We also thank all participants for this study and their families.

\section{STATEMENT OF FINANCIAL SUPPORT}

US Department of Agriculture, Agricultural Research Service (USDA/ARS 6251-51000-005-02).

Disclosure: There is no conflict of interest to disclose.

\section{REFERENCES}

1. Neville MC, Anderson SM, McManaman JL, et al. Lactation and neonatal nutrition: defining and refining the critical questions. J Mammary Gland Biol Neoplasia 2012;17:167-88.

2. Smith MM, Durkin M, Hinton VJ, Bellinger D, Kuhn L. Influence of breastfeeding on cognitive outcomes at age 6-8 years: follow-up of very low birth weight infants. Am J Epidemiol 2003;158:1075-82.

3. Lucas A, Morley R, Cole TJ, Lister G, Leeson-Payne C. Breast milk and subsequent intelligence quotient in children born preterm. Lancet 1992;339:261-4.

4. Georgieff MK. Nutrition and the developing brain: nutrient priorities and measurement. Am J Clin Nutr 2007;85:614S-20S.

5. Andres A, Cleves MA, Bellando JB, Pivik RT, Casey PH, Badger TM. Developmental status of 1-year-old infants fed breast milk, cow's milk formula, or soy formula. Pediatrics 2012;129:1134-40. 
6. Kramer MS, Aboud F, Mironova E, et al.; Promotion of Breastfeeding Intervention Trial (PROBIT) Study Group. Breastfeeding and child cognitive development: new evidence from a large randomized trial. Arch Gen Psychiatry 2008;65:578-84.

7. Oddy WH, Li J, Whitehouse AJ, Zubrick SR, Malacova E. Breastfeeding duration and academic achievement at 10 years. Pediatrics 2011;127:e137-45.

8. Quigley MA, Hockley C, Carson C, Kelly Y, Renfrew MJ, Sacker A. Breastfeeding is associated with improved child cognitive development: a population-based cohort study. J Pediatr 2012;160:25-32.

9. Mortensen EL, Michaelsen KF, Sanders SA, Reinisch JM. The association between duration of breastfeeding and adult intelligence. JAMA 2002;287:2365-71.

10. Ferreira Hda S, Xavier Júnior AF, de Assunção ML, Dos Santos EA, Horta BL. Effect of breastfeeding on head circumference of children from impoverished communities. Breastfeed Med 2013;8:294-301.

11. Herba CM, Roza S, Govaert P, et al. Breastfeeding and early brain development: the Generation R study. Matern Child Nutr 2013;9:332-49.

12. Isaacs EB, Fischl BR, Quinn BT, Chong WK, Gadian DG, Lucas A. Impact of breast milk on intelligence quotient, brain size, and white matter development. Pediatr Res 2010;67:357-62.

13. Kafouri S, Kramer M, Leonard G, et al. Breastfeeding and brain structure in adolescence. Int J Epidemiol 2013;42:150-9.

14. Isaacs EB. Neuroimaging, a new tool for investigating the effects of early diet on cognitive and brain development. Front Hum Neurosci 2013;7:445.

15. Ashtari M, Kumra S, Bhaskar SL, et al. Attention-deficit/hyperactivity disorder: a preliminary diffusion tensor imaging study. Biol Psychiatry 2005;57:448-55.

16. Barnea-Goraly N, Kwon H, Menon V, Eliez S, Lotspeich L, Reiss AL. White matter structure in autism: preliminary evidence from diffusion tensor imaging. Biol Psychiatry 2004;55:323-6.

17. Cullen KR, Klimes-Dougan B, Muetzel R, et al. Altered white matter microstructure in adolescents with major depression: a preliminary study. J Am Acad Child Adolesc Psychiatry 2010;49:173-83.e1.

18. Rimrodt SL, Peterson DJ, Denckla MB, Kaufmann WE, Cutting LE. White matter microstructural differences linked to left perisylvian language network in children with dyslexia. Cortex 2010;46:739-49.

19. Mabbott DJ, Rovet J, Noseworthy MD, Smith ML, Rockel C. The relations between white matter and declarative memory in older children and adolescents. Brain Res 2009;1294:80-90.

20. Qiu D, Tan LH, Zhou K, Khong PL. Diffusion tensor imaging of normal white matter maturation from late childhood to young adulthood: voxelwise evaluation of mean diffusivity, fractional anisotropy, radial and axial diffusivities, and correlation with reading development. Neuroimage 2008;41:223-32.

21. Schmithorst VJ, Wilke M, Dardzinski BJ, Holland SK. Cognitive functions correlate with white matter architecture in a normal pediatric population: a diffusion tensor MRI study. Hum Brain Mapp 2005;26:139-47.

22. Nakayama N, Okumura A, Shinoda J, et al. Evidence for white matter disruption in traumatic brain injury without macroscopic lesions. J Neurol Neurosurg Psychiatr 2006;77:850-5.

23. Werring DJ, Clark CA, Barker GJ, Thompson AJ, Miller DH. Diffusion tensor imaging of lesions and normal-appearing white matter in multiple sclerosis. Neurology 1999;52:1626-32.
24. Smith SM, Jenkinson M, Johansen-Berg H, et al. Tract-based spatial statistics: voxelwise analysis of multi-subject diffusion data. Neuroimage 2006;31:1487-505.

25. Choi J, Jeong B, Polcari A, Rohan ML, Teicher MH. Reduced fractional anisotropy in the visual limbic pathway of young adults witnessing domestic violence in childhood. Neuroimage 2012;59:1071-9.

26. Liu T, Zhou Y, Chen L, et al. Structural insights into cellulolytic and chitinolytic enzymes revealing crucial residues of insect $\mathrm{B}$ - $\mathrm{N}$-acetyl-D-hexosaminidase. PLoS ONE 2012;7:e52225.

27. Deoni SC, Dean DC 3rd, Piryatinsky I, et al. Breastfeeding and early white matter development: A cross-sectional study. Neuroimage 2013;82:77-86.

28. Simmonds DJ, Hallquist MN, Asato M, Luna B. Developmental stages and sex differences of white matter and behavioral development through adolescence: a longitudinal diffusion tensor imaging (DTI) study. Neuroimage 2014;92:356-68.

29. Dick AS, Tremblay P. Beyond the arcuate fasciculus: consensus and controversy in the connectional anatomy of language. Brain 2012;135 (Pt 12):3529-50.

30. Takahashi M, Iwamoto K, Fukatsu H, Naganawa S, Iidaka T, Ozaki N. White matter microstructure of the cingulum and cerebellar peduncle is related to sustained attention and working memory: a diffusion tensor imaging study. Neurosci Lett 2010;477:72-6.

31. Chaddock-Heyman L, Erickson KI, Voss MW, et al. White matter microstructure is associated with cognitive control in children. Biol Psychol 2013;94:109-15.

32. Sullivan EV, Zahr NM, Rohlfing T, Pfefferbaum A. Fiber tracking functionally distinct components of the internal capsule. Neuropsychologia 2010;48:4155-63.

33. Kang X, Herron TJ, Woods DL. Regional variation, hemispheric asymmetries and gender differences in pericortical white matter. Neuroimage 2011;56:2011-23.

34. Nagahara K, Dobashi K, Itabashi K. Feeding choice has a gender-associated effect on infant growth. Pediatr Int 2013;55:481-7.

35. Lucas A, Morley R, Cole TJ. Randomised trial of early diet in preterm babies and later intelligence quotient. BMJ 1998;317:1481-7.

36. Feldman HM, Lee ES, Yeatman JD, Yeom KW. Language and reading skills in school-aged children and adolescents born preterm are associated with white matter properties on diffusion tensor imaging. Neuropsychologia 2012;50:3348-62.

37. Demerens C, Stankoff B, Logak M, et al. Induction of myelination in the central nervous system by electrical activity. Proc Natl Acad Sci USA 1996;93:9887-92.

38. Stevens B, Porta S, Haak LL, Gallo V, Fields RD. Adenosine: a neuronglial transmitter promoting myelination in the CNS in response to action potentials. Neuron 2002;36:855-68.

39. Jing H, Gilchrist JM, Badger TM, Pivik RT. A longitudinal study of differences in electroencephalographic activity among breastfed, milk formulafed, and soy formula-fed infants during the first year of life. Early Hum Dev 2010;86:119-25.

40. Kodama Y, Kikusui T, Takeuchi Y, Mori Y. Effects of early weaning on anxiety and prefrontal cortical and hippocampal myelination in male and female Wistar rats. Dev Psychobiol 2008;50:332-42. 